\title{
BMJ Open Intravascular device administration sets: replacement after standard versus prolonged use in hospitalised patients - a study protocol for a randomised controlled trial (The RSVP Trial)
}

Claire M Rickard, ${ }^{1,2,3,4}$ Nicole M Marsh, ${ }^{1,2}$ Joan Webster, ${ }^{1,2}$ Nicole C Gavin, ${ }^{1,2}$ Matthew R McGrail, ${ }^{5}$ Emily Larsen, ${ }^{1,2}$ Amanda Corley, ${ }^{1,4}$ Debbie Long, ${ }^{1,6}$ John R Gowardman, ${ }^{1,2}$ Marghie Murgo, ${ }^{7,8}$ John F Fraser, ${ }^{1,4}$ Raymond J Chan, ${ }^{1,2,9}$ Marianne C Wallis, ${ }^{1,10}$ Jeanine Young, ${ }^{6,10}$ David McMillan, ${ }^{11}$ Li Zhang, ${ }^{1}$ Md Abu Choudhury, ${ }^{1,2}$ Nicholas Graves, ${ }^{9}$ E Geoffrey Playford ${ }^{1,3}$

To cite: Rickard CM, Marsh NM, Webster J, et al. Intravascular device administration sets: replacement after standard versus prolonged use in hospitalised patients-a study protocol for a randomised controlled trial (The RSVP Trial). BMJ Open 2015;5:e007257. doi:10.1136/bmjopen-2014007257

- Prepublication history and additional material is available. To view please visit the journal (http://dx.doi.org/ 10.1136/bmjopen-2014007257).

Received 20 November 2014 Revised 8 December 2014 Accepted 9 December 2014

CrossMark

For numbered affiliations see end of article.

Correspondence to Professor Claire M Rickard; c.rickard@griffith.edu.au

\section{ABSTRACT}

Introduction: Vascular access devices (VADs), such as peripheral or central venous catheters, are vital across all medical and surgical specialties. To allow therapy or haemodynamic monitoring, VADs frequently require administration sets (AS) composed of infusion tubing, fluid containers, pressure-monitoring transducers and/or burettes. While VADs are replaced only when necessary, AS are routinely replaced every 3-4 days in the belief that this reduces infectious complications. Strong evidence supports AS use up to 4 days, but there is less evidence for AS use beyond 4 days. AS replacement twice weekly increases hospital costs and workload.

Methods and analysis: This is a pragmatic, multicentre, randomised controlled trial (RCT) of equivalence design comparing AS replacement at 4 (control) versus 7 (experimental) days. Randomisation is stratified by site and device, centrally allocated and concealed until enrolment. 6554 adult/paediatric patients with a central venous catheter, peripherally inserted central catheter or peripheral arterial catheter will be enrolled over 4 years. The primary outcome is VADrelated bloodstream infection (BSI) and secondary outcomes are VAD colonisation, AS colonisation, allcause BSI, all-cause mortality, number of AS per patient, VAD time in situ and costs. Relative incidence rates of VAD-BSI per 100 devices and hazard rates per 1000 device days (95\% Cls) will summarise the impact of 7day relative to 4-day AS use and test equivalence. KaplanMeier survival curves (with log rank Mantel-Cox test) will compare VAD-BSI over time. Appropriate parametric or non-parametric techniques will be used to compare secondary end points. $p$ Values of $<0.05$ will be considered significant.

Ethics and dissemination: Relevant ethical approvals have been received. CONSORT Statement recommendations will be used to guide preparation of any publication. Results will be presented at relevant conferences and sent to the major organisations with clinical practice guidelines for VAD care.
Trial registration number: Australian New Zealand Clinical Trial Registry (ACTRN 12610000505000).

\section{INTRODUCTION}

Vascular access devices (VADs) are small hollow catheters inserted into a vein or artery. VAD insertion is the most common invasive healthcare procedure with approximately 14 million VADs used in Australia each year and billions throughout the world. ${ }^{1}$ VADs are inserted either centrally (eg, central venous catheter $(\mathrm{CVC})$ ) or peripherally (eg, peripherally inserted central venous catheter (PICC)) for haemodynamic monitoring and the delivery of intravascular fluids, nutrition and medications. VADs also include peripheral arterial catheters (PAC) used predominantly in the operating theatre and critical care units to provide continuous blood pressure monitoring and access for repeated blood sampling. In order to deliver many therapies, VADs require connection to an administration set (AS). The AS refers to infusion systems comprised of some or all of tubing, fluid containers, pressure monitoring transducers, blood sampling ports, measuring burettes, extension tubing and needleless connectors. VADs break the natural defence barrier of the skin and are the single most important cause of healthcare-acquired bloodstream infection (BSI). About 250 000-500 000 VAD-BSIs occur each year in the USA alone. ${ }^{2}$ VAD-BSIs are associated with increased mortality and substantially increase hospital stay and 
treatment costs by up to 20 days and US $\$ 56000 /$ episode, respectively. ${ }^{2}$

Clinicians traditionally limit the duration of AS use by routinely replacing AS at regular intervals. That is, the VAD may be used for a week or more, whereas the AS is routinely disconnected, discarded and replaced every 3-4 days. The practice of routinely changing AS is not based on strong evidence of efficacy. Prior to the 1970s, AS were used until no longer required. ${ }^{3}{ }^{4}$ Routine AS replacement developed as a response to the 1970-1971 USA epidemic of VAD-BSI; this epidemic was later traced to manufacturer-contaminated intravenous fluid. $^{3} 5$ Despite the unrelated nature of this cause to the duration of AS use, $24 \mathrm{~h}$ routine replacement of AS was advocated and universally implemented at that time. ${ }^{6} 7$ The policy of routine replacement has continued since.

\section{VAD AS replacement at 24-96 h}

Each AS replacement involves significant nursing time and costs up to $\$$ A300 (2004) for one VAD AS change in equipment alone. ${ }^{8}$ Since 1971, the length of time between AS changes has gradually been extended. Several randomised controlled trials (RCTs) compared time frames such as 24 versus $48 \mathrm{~h}$ or 24 versus $72 \mathrm{~h}$. A Cochrane Collaboration systematic review and meta-analysis of 16 RCTs included 5001 patients and AS use of 24 to $\geq 96 \mathrm{~h}^{9}{ }^{9}$ No significant difference was found in VAD-BSI with longer periods of AS use. In addition, no significant effect was found on any of the device colonisation, infusate colonisation, infusaterelated BSI or all-cause BSI. There was some evidence that less frequent AS replacement increased mortality rate in neonates (RR 1.84; 95\% CI 1.00 to 3.66). This was based on two studies of 24 versus $48 \mathrm{~h}$ AS replacement ${ }^{10}$ and 24 versus $72 \mathrm{~h}$ replacement, ${ }^{11}$ with $96 \%$ of the statistical weight coming from the latter study which was at risk of bias from a suboptimal randomisation technique $(10 \%$ of sample were rerandomised).

\section{VAD AS use beyond $96 \mathrm{~h}$}

The Cochrane Review found inadequate trials to investigate AS use beyond $96 \mathrm{~h}^{9}{ }^{9}$ One RCT (512 patients) compared 3-day with 'between 4-day and 7-day' use and found no significant difference in infusate-related BSI. ${ }^{12}$ An unpublished conference abstract reported another RCT of 769 patients with AS replaced at 72 or $120 \mathrm{~h}$, with no between-group differences in catheter colonisation or VAD-BSI. ${ }^{13}$ Jakobsen et $a l^{14}$ undertook a five-group RCT with AS changed at 24, 48, 72, 96 or $120 \mathrm{~h}$ and found no difference in catheter colonisation. Luskin $e t a l^{15}$ randomised 112 patients to have AS changes at 48 , or $96 \mathrm{~h}$ to $192 \mathrm{~h}$ and reported no difference in infusate colonisation, infusate-related BSI or device colonisation. In vitro work found AS retained accuracy for volume delivery and good physical condition after 7 days continuous use. ${ }^{16}$ Clinical studies have demonstrated identical rates of VAD-BSI and no significant difference in VAD colonisation when AS were replaced at 3-4 days or 7 days, ${ }^{8}{ }^{17}$ with substantial cost savings in AS and nursing time, ${ }^{17}$ and no relationship between infusate colonisation or VAD-BSI and time. ${ }^{18}$

\section{Clinical practice guidelines and expert recommendations}

Joint guidelines from Healthcare Epidemiology and the Infectious Diseases Societies of America cite the Cochrane review ${ }^{9}$ and recommend replacing AS at 'not longer than $96 \mathrm{~h}$ intervals'. ${ }^{19} 20$ Australia's Infection Prevention Guidelines also recommended maximum 4-day use. ${ }^{21}$ The Centers for Disease Control and Prevention (CDC) 'Guidelines for the Prevention of VAD Related Infections' ${ }^{22}$ recommends changing AS 'no more frequently than every $96 \mathrm{~h}$, but at least every 7 days'. This highlights the uncertainty of relative risk between these two time frames. All AS guidelines exclude high growth media of lipid-based and blood-based AS infusions which are discarded within $24 \mathrm{~h}$.

\section{Theoretical framework for AS replacement}

The theoretical principle behind routine AS replacement is that AS are initially sterile and become contaminated through clinical use, but if removed at set intervals, BSI is prevented. ${ }^{23}$ Limiting AS use to 4 days presumes microbes within sets are not problematic before day 4 and ignores the potential for immediate microbial contamination when staff inject medicine or fluids. It seems likely that if an AS is contaminated, virulent pathogens would cause significant growth and patient infection within a shorter time; thus, fourth day replacement would have no effect on pathogens already infused. ${ }^{1524}$

Routine AS replacement itself provides an opportunity for contamination, as it breaks a closed circuit, and this suggests it should actually be avoided. ${ }^{25}$ Increased occasions of AS manipulations are known to increase infection, yet routine replacement causes extra handling of the hub and AS. ${ }^{6}{ }^{26}$ Poor aseptic technique in accessing the AS is not uncommon ${ }^{27}$ and would likely over-ride any benefit of routine AS replacement. Further, AS replacement does not replace the hub component of the VAD itself, which is known to be the main reservoir for infection from VADs after the first week. ${ }^{28}$ The current incongruous situation is that VADs are used for extended periods and only removed for VAD-BSI or at completion of treatment, yet the AS are disconnected and replaced twice each week.

VADs are vital and ubiquitous medical devices, with billions used around the world each year. Consequently, reliable evidence that informs the safe, effective use of these devices is important to patients, nurses, doctors and the community. The aim of the study is to compare the impact of VAD AS replacement at 4 versus 7 days on infective, clinical and cost outcomes. The primary hypothesis is that there will be equivalence in the incidence of VAD-BSIs between patients assigned to AS replacement at 4 versus 7 days. We also hypothesise that there will be equivalence between groups in the incidence of: (1) AS colonisation, (2) hub colonisation, (3) VAD colonisation, (4) all-cause $\mathrm{BSI}$ rate, (5) VAD time in situ, and (6) all-cause mortality; 
and that patients assigned to 4-day replacement will have significantly higher: (1) number of AS used and (2) associated healthcare costs.

\section{METHODS AND ANALYSIS}

The study is a pragmatic, multicentre, RCT of equivalence design. Adult and paediatric patients with a CVC, PICC or PAC will be enrolled. The experimental group will have AS routinely replaced every 7 days and the control group every 4 days. There will be a 4 -year recruitment period of 6554 patients. The protocol is V.3 dated 17 February 2014.

\section{Outcome measures}

\section{Primary outcome}

\section{VAD-BSI}

1. Primary bacteraemia/fungaemia with $\geq 1$ positive blood culture from a peripheral vein with no other identifiable source for the BSI other than the VAD, plus a positive semiquantitative $(>15$ colony forming units (CFU)) device culture, with the same organism (species and antibiogram) isolated from the device tip and the blood; ${ }^{22}$

or

2. Two blood cultures (one from a VAD and one from a peripheral vein) that meet the VAD-BSI criteria for differential time to positivity (DTP) (growth of the same microbe from hub-drawn blood at least $2 \mathrm{~h}$ before growth from the peripheral blood). ${ }^{29}$

Criterion 1 is generally used for diagnosis in short-term catheters (CVCs and PACs) where the device is commonly removed when infection is suspected. Criteria 2 is generally used for diagnosis in long-term CVCs and PICCs where the device is often left in situ when infection is suspected. The original study protocol (as listed in the trial registry when started) included additional options for diagnosis of the Primary Outcome using blood and tip culture results using quantitative laboratory culture methods. These were deleted at a later stage since none of the study sites use quantitative blood or tip culture techniques in their hospital laboratories.

\section{Secondary outcomes}

A. VAD colonisation: Growth of $>15$ CFU from distal segment of VAD tip on removal, using the semiquantitative culture method. ${ }^{30}$ The original trial proto$\mathrm{col}$ in the registry also listed an option for diagnosis using quantitative culture results. This option was deleted at a later stage since none of the study sites use quantitative blood or tip culture techniques in their hospital laboratories.

B. AS colonisation: Growth of $\geq 10^{3} \mathrm{CFU} / \mathrm{mL}$ from the AS using quantitative culture. ${ }^{32} 33$ This end point is performed in the research laboratory (not the hospital laboratory) where quantitative methods are available.

C. All-cause BSI: A bacteraemia or fungaemia obtained from a peripheral vein and taken while the VAD is in situ, or within $48 \mathrm{~h}$ of removal. ${ }^{31}$
D. All-cause mortality: Mortality at hospital discharge.

E. Time in situ: Hours of device use from insertion to removal. $^{31}$

F. AS per patient: Number of AS used from the time of study entry to discharge from hospitalisation.

G. Costs: AS consumable prices, staff time and treatment costs for VAD-BSI. ${ }^{34}$

\section{Setting and sample}

The trial will be undertaken at eight major hospitals in Australia. The study hospitals together have over 500000 separations per annum, and cover the full range of medical and surgical specialties. Adult and paediatric patients admitted to acute medical, surgical, oncology, haematology and critical care wards will be screened. Inclusion criteria are informed written or documented verbal consent (if need for consent not waived by the Human Research Ethics Committee (HREC) for that hospital), central venous (including PICCs) and/or peripheral arterial VAD in situ with AS, VAD in situ for $>24 \mathrm{~h}$, and VAD scheduled/expected use $\geq 7$ days. Exclusion criteria are BSI within previous $48 \mathrm{~h}$, planned removal of device $\leq 24 \mathrm{~h}$, VAD in situ $>96 \mathrm{~h}$, and original AS already routinely replaced.

\section{Sample size}

Sample size calculations determined that 1371 CVCs, 1268 PICCs and 340 PACs are required per group (total VADs 2979 per group) for $>90 \%$ power at $\mathrm{p}=0.05$ (PASS, Power Analysis and Sample Size system, NCSS, Utah, USA). The proportion of VAD-BSI in the reference (control) group is estimated to be $2.6 \%, 2.4 \%$ and $0.8 \%$ for CVC, PICC and $\mathrm{PAC}$, respectively, taken from a metasynthesis of VAD-BSI rates in $>2$ million VADs-using identical definitions as in our study. ${ }^{22}$ A cumulative approach was used for the three device types (CVC, PICC, PAC). To allow for potential attrition, a further $10 \%$ (298 VADs) will be recruited per group. An equivalence hypothesis was used for CVCs and PICCs with $\pm 2 \%$ limits. As PAC rates are already extremely low $(0.8 \%)$, a non-inferiority approach was used ( $+2 \%$ bound). The $2 \%$ margin reflects all previous welldesigned RCTs on the topic of AS duration, with absolute differences between groups of $-1.7 \%$ to $1.3 \%$. The two prior RCTs ${ }^{8}{ }^{12}$ that tested 3-day/4-day use with 7-day use observed identical absolute rates of VAD-BSI in the two groups. Raad $e t a l^{12}$ also used $2 \%$ bounds for equivalence. Based on our pilot and the Cochrane review, ${ }^{89}$ we consider $>2 \%$ difference in the primary end point between groups to be very unlikely.

\section{Recruitment and randomisation}

The medical, surgical, haematology-oncology wards and intensive care units (ICU) will be screened at least 3 days each week by dedicated research nurses and all eligible patients (or their representative) will be approached for informed consent (except in hospitals where the requirement for consent has been waived by the local HREC). Patients will be randomly assigned, 
using computer-generated random assignment in a $1: 1$ ratio, to either the 4-day AS change group or 7-day AS change group. This will be stratified by device type (short-term CVC, long-term CVC, PICC or PAC), acuity (ICU and non-ICU), and hospital site to ensure equal distribution of patient and therapy types between groups. As randomisation will be conducted via a centralised computer randomisation service, allocation will be concealed until the point of randomisation.

\section{Replacement and care of AS}

The experimental group will have all AS changed on day 7 , and then weekly, by clinical staff. The control group will have AS changed by clinical staff every 4 days. In both groups, additional AS reconfiguration will occur if clinically indicated due to treatment addition/completion, VAD removal or AS malfunction. AS configurations, number, timing and reason for AS changes will be documented. VADs will be removed at the decision of the treating clinician. Clinical staff will be responsible for the insertion and care of VADs and AS following CDC Guidelines, including discard of any AS used for chemotherapy immediately after use and blood-based or lipid-based products within 12 and $24 \mathrm{~h}$, respectively. ${ }^{31}$ Protocol adherence will be monitored by the research nurses, with breaks in the protocol documented. Once the VAD is removed (even if replaced by guidewire into the same vein), or continuous infusions have been ceased for the three study visits, the patient has completed the study (with the exception of the $48 \mathrm{~h}$ follow-up).

\section{Microbiological testing and end points}

Blood and VAD tip cultures will be taken by clinical staff on suspicion of infection as per usual hospital and pathology department protocols. It is well validated that it is unnecessary to culture non-symptomatic patients' samples routinely, as almost identical rates of VAD-BSI are achieved with culture only on clinical suspicion. ${ }^{2}$ All patients with a BSI while the VAD is in situ $(+48 \mathrm{~h}$ postremoval) will have a standardised data collection sheet (that includes all data necessary for VAD-BSI diagnosis as per the CDC definitions but does not reveal the study group) prepared by the research nurses. This data will then be reviewed by one of the blinded investigators (EGP) who is an infectious diseases specialist familiar with the diagnosis and treatment of VAD-BSI. This investigator will assign the primary end point of VAD-BSI and nominate the likely source (VAD, AS, skin or haematogenous seeding). The blinded hospital microbiology staff will assign the secondary end points of BSI and device colonisation, using objective measures and techniques that are routine daily procedures. ${ }^{30}$ For the secondary end points of hub and AS colonisation, a random subset of 325 patients per group whose VAD are removed for suspected VAD-BSI and 325 patients per group without suspected VAD-BSI (total $20 \%$ of sample) will have AS samples cultured. ${ }^{35}$ The specimens will be processed by scientists with expertise in such bacterial counts and identification. ${ }^{32} 33$ These end points will be of interest regarding potential pathogenic impact of AS replacement timing. It would be unfeasible and prohibitively expensive to perform these for the whole sample and also unnecessary, given that our primary interest is the effectiveness of the intervention to prevent clinical infection.

\section{Blinding}

This trial is blinded for the primary end point of VAD-BSI and for the secondary end points of AS, device and hub colonisation. These are performed by blinded infectious disease and microbiologists. AS replacement procedures and the time frames will not be possible to conceal from patients, research nurses or clinical staff. However, as they are not assessing the primary end point this will not introduce bias.

\section{Data collection}

The research nurses, all of whom have had study-specific database training, will directly enter data in the clinical areas using hand-held password-protected electronic devices with a purpose-built Access database and form-based interface or using REDCap (project-redcap. org). This technology is well established in our group. All data is de-identified at this point and only identifiable within the database by specific study number. The master list of participants will be kept separate to the study database in a different password-protected computer programme. Baseline data will include age, gender, diagnostic group, severity of infection risk and immunosuppression status. ${ }^{36-38}$ Other data collected will be the concurrent infection, antibiotic therapy, type of infusate and additives, VAD type, insertion site, inserter, VAD dwell time, AS hang time, reason for $\mathrm{VAD}$ and $\mathrm{AS}$ removal, and mortality status. ${ }^{6} 1937$ AS configuration at the time of AS recruitment and at each replacement will be noted. Microbiological data will include BSI, VAD and other cultures taken during AS use and for $48 \mathrm{~h}$ postremoval if patients are still in hospital. Some patients in each group will cease VAD therapy for clinical reasons $<96 \mathrm{~h}$ (eg, deceased). Outcome data will be collected for these cases and included in the final analysis.

\section{Data analysis}

Intention to treat analysis will be used and the patient is the unit of measurement (patient randomised once but may have multiple VADs and AS). Relative incidence rates of VAD-BSI per 100 devices and hazard rates per 1000 device days with $95 \%$ CIs will summarise the impact of the 7-day relative to 4-day AS use and test equivalence for CVCs, PICCs and PACs. Kaplan-Meier survival curves (with log rank Mantel-Cox test) will compare VAD-BSI rates over time. Subgroup analysis will be stratified by VAD type. Secondary end points will be compared between groups using parametric or non-parametric techniques appropriate to level of measurement. Secondary analysis using multivariate regression (Cox) 
will test the effect of variables associated with VAD-BSI risk on the outcome: lipids, parenteral nutrition, antibiotics, length of stay, diagnostic group, initial versus subsequent VADs, age, gender, VAD-BSI in a preceding catheter, site of VAD placement, inserter and patient risk category. All possible attempts will be made to collect the primary end point. Missing data will be modelled for bestcase and worst-case outcomes to assess any potential effect on overall results and a per-protocol analysis will assess the effect of protocol violations. $p$ Values of $<0.05$ will be considered significant. We do not expect effect sizes to vary between sites; however, intersite variability will be assessed and if necessary, adjustment is made in the model, with consideration of potential institutional differences in patient variables.

\section{Estimating cost parameters}

Procedure costs will be measured by assessing the staff time associated with AS change, and the type and volume of equipment used. ${ }^{32} 33$ The economic opportunity costs of workers' time will be valued by national wage rates to include full costs of employment; costs of equipment will be valued based on standard contracts between state health services and commercial manufacturers. Mean costs of 7-day and 4-day changes will be compared to identify the likely cost savings of a decision to make 7-day changes standard practice.

\section{Time frame and feasibility}

It is expected that, with staggered site start-ups, recruitment will be 4 years, 6 months. We will implement trialspecific education to ensure clinical staff support for the study and protocol adherence. To test if the 4-day and 7-day replacement are equivalent, it is crucial that adequate numbers of AS are used beyond this point. It is expected that a small number of VADs in both groups will be removed before scheduled AS replacement; this reflects the real world of clinical practice and will not limit generalisability.

\section{MONITORING, ETHICS AND DISSEMINATION OF RESULTS Monitoring}

Study managers (NMM and ER) who report to the chief investigator (CMR) and are employed by the sponsor (Griffith University), provide initial training and ongoing support to the research nurses and site investigators, and monitor data quality and site processes, at least monthly.

\section{Ethical and safety considerations}

Both 4-day and 7-day AS use is within the recommendations of the CDC and the Cochrane Collaboration. Seven-day AS use has been used in some hospitals since the completion of our pilot study, and other reports also support its safety. 8121718 Participants or representatives may give either written or documented verbal consent or the consent may be waived depending on processes acceptable at each hospital under the relevant State legislation. For paediatrics, as per the National Statement on Ethical Conduct in Human Research, consent will be sought from both the child, if sufficiently competent, and parents. As part of providing consent, participants also consent to the data and specimens being used for subsequent studies relevant to infection prevention and VAD care. Serious adverse events (SAE) of mortality or ICU admission while on the trial will be monitored and reported to the Human Research Ethics Committee. A data safety monitoring committee (DSMC) of two biostatisticians and two medically qualified researchers (independent of the study) will review the primary end point and SAEs after each 1000 cases. The DSMC will inform the investigators if (1) based on the VAD-BSI rate, the sample size needs to be significantly recalculated or (2) the study needs to be stopped because of a statistically higher VAD-BSI or SAE rate favouring either group.

\section{Dissemination}

Neither the sponsor nor funder will be involved in data analysis or reporting. Data will be analysed by MRM the study statistician. Only the research team will have access to the study data; data generated from the trial will be available for inspection by the HREC and funders on request. The prevention of healthcare-associated infections and responsible use of the health dollar are of high interest to all stakeholders, including the community. We will present results locally, and at relevant local and international meetings of the infection control and vascular access specialty groups, and a media release will be circulated. The investigators will be available for interviews in the community/health media and we will supply results to organisations that publish clinical practice guidelines for the care of patients with VADs. The study will be published in a peer-reviewed healthcare journal. Authorship will be consistent with the International Committee of Medical Journal Editors' criteria, and will be undertaken by the investigators without the use of professional writers. The results will have international application and should be rapidly translated into clinical policies. During the study, monthly investigator meetings will be held at one of the participating hospitals, with remote sites joining by teleconference/videoconference. These meetings are a forum for tracking recruitment and communicating important protocol modificants or clarifications. These issues are also followed up via email or telephone calls to the Local Site Investigators/Study Nurses by the Study Managers (NMM, EL). The study managers along with the chief investigator (CMR) also organise regular updates and annual reports to the Human Research Ethics Committees, local hospital Research Governance Officers, the granting bodies and the trial registry.

\section{TRIAL STATUS}

Recruitment of patients to the study is ongoing. The first patient was recruited in May 2011 and it is expected 
that recruitment will be completed in December 2015. Steady recruitment has been maintained through regular site visits by the project manager, monthly study meetings of all participating sites and continued education to both clinical and study staff at each site. Additional and replacement study sites have been added over the course of the trial to increase participant enrolment and/or to address the resignation of the local site principle investigator, where relevant.

\section{DISCUSSION}

While good evidence exists to support AS use up to 4 days, ${ }^{9}$ there are minimal data about use beyond this time. Approximately 14 million VADs are used each year in Australia alone and nurses care for these devices daily. ${ }^{1}$ Changing from 4-day to 7-day AS replacement saved US $\$ 7425$ per year per ward in a US study. ${ }^{17}$ In Australia, each AS replacement costs up to $\$$ A300 (2004 estimate) in equipment and about 0.5 nursing hours. ${ }^{8}$ If 7-day AS use is adopted and even if four million VADs avoided one AS replacement at $\$ 250$, then $\$ A 1$ billion and 2 million nursing hours would be saved each year. This would also incur significant reductions in plastic waste. Evidence to date has favoured the adult intensivecare setting and it is vital that further knowledge is gathered across the spectrum of VAD use in medical, surgical, oncology and haematology units, as well as paediatric patients. This study will support or refute the practice of restricting AS use to 4 days and if the safety of 7-day use is confirmed, will change practice worldwide.

\section{Author affiliations}

${ }^{1}$ NHMRC Centre of Research Excellence in Nursing (NCREN), Centre for Health Practice Innovation—Griffith Health Institute, Griffith University, Brisbane, Australia

${ }^{2}$ Royal Brisbane and Women's Hospital, Brisbane, Australia

${ }^{3}$ Infection Management Services, Princess Alexandra Hospital, Brisbane, Australia

${ }^{4}$ Critical Care Research Group, The Prince Charles Hospital and University of Queensland, Brisbane, Australia

${ }^{5}$ School of Rural Health, Monash University, Churchill, Australia

${ }^{6}$ Lady Cilento Children's Hospital, Brisbane, Australia

${ }^{7}$ Clinical Excellence Commission, Sydney, Australia

${ }^{8}$ Royal Prince Alfred Hospital, Sydney, Australia

${ }^{9}$ Institute of Health and Biomedical Innovation, Queensland University of Technology, Brisbane, Australia

${ }^{10}$ School of Nursing and Midwifery, University of the Sunshine Coast, Maroochydore, Australia

${ }^{11}$ Inflammation and Healing Research Cluster, School of Health and Sport Sciences, University of the Sunshine Coast, Maroochydore, Australia

Twitter Follow Claire Rickard at @avatar_grp, Emily Larsen at $@$ EmilyLarsenAU, Margherita Murgo at @mlase and David McMillan at @DrDaveMacca

Acknowledgements The authors would like to thank Professor Michael Whitby, Princess Alexandra Hospital, for assistance with funding applications; The National Health and Medical Research Council, The Royal Brisbane and Women's Hospital Foundation, the Cancer Nurses' Society of Australia, the Gold Coast Hospital Foundation, Griffith University and the Australian College of Critical Care Nurses (Queensland) for research grant funding.

Contributors CMR conceived the study, wrote grant, developed protocol and funding application, wrote the first draft of manuscript and approved the final draft. NMM and NCG made proposal development, grant application, managed the study, reviewed manuscript and approved the final draft. JW made proposal development, grant application, oversight data collection adult setting, reviewed manuscript and approved the final draft. MRM contributed to statistical methods, proposal development, grant application, reviewed the manuscript and approved the final draft. EL contributed in data collection adult setting, assistance with study management and primary end point assignment, reviewed the manuscript and approved the final draft. AC contributed in grant application, data collection adult setting, prepared and reviewed the manuscript, and approved the final draft. DL and JY contributed in grant application, oversight data collection paediatric setting, reviewed the manuscript and approved the final draft. JRG, JFF and RJC contributed in grant application, oversight data collection adult setting, reviewed the manuscript and approved the final draft. MM contributed in oversight and data collection adult setting, reviewed the manuscript and approved the final draft. MCW contributed to grant application, reviewed the manuscript and approved the final draft. DM contributed to microbiological methods, grant application, oversight microbiological data, reviewed the manuscript and approved the final draft. LZ contributed to microbiological methods, grant application, reviewed the manuscript and approved the final draft. MAC contributed to microbiological methods, reviewed the manuscript and approved the final draft. NG contributed to health economic methods, grant application, reviewed the manuscript and approved the final draft. EGP contributed in proposal development, blinded primary end point rating, data collection adult setting, reviewed the manuscript and approved the final draft.

Funding The sponsor is Griffith University who is the employer of the principal investigator (CMR). This work is supported by competitive grants from the Australian National Health and Medical Research Council (APP1008428), The Royal Brisbane and Women's Hospital Foundation, the Cancer Nurses' Society of Australia and the Australian College of Critical Care Nurses (Queensland)

Competing interests CMR's institution has received research funding and speaker fees unrelated to this trial on her behalf from commercial suppliers of vascular access products: 3M, BBraun, BD, Bard, Carefusion, Centurion and Mayo. NMM's institution has received research funding and speaker fees unrelated to this trial on her behalf from commercial suppliers of vascular access products 3M and BD. RJC's institution has received research funding and speaker fees unrelated to this trial on his behalf from a commercial supplier of vascular access products, Centurion. No commercial entity had any role in the design or undertaking of this study.

Ethics approval Human Research Ethics Committee approval has been granted by Queensland Health (HREC/13/QRCH/185), Liverpool Hospital (HREC/12/RPAH/337), Sir Charles Gairdner Hospital (SCGH/PMH/RPH/HREC/ 2012-183) and Griffith University (NRS/27/10/HREC), and the trial is registered with the Australian New Zealand Clinical Trials Registry (ACTRN12610000505000).

Provenance and peer review Not commissioned; internally peer reviewed.

Open Access This is an Open Access article distributed in accordance with the Creative Commons Attribution Non Commercial (CC BY-NC 4.0) license, which permits others to distribute, remix, adapt, build upon this work noncommercially, and license their derivative works on different terms, provided the original work is properly cited and the use is non-commercial. See: http:// creativecommons.org/licenses/by-nc/4.0/

\section{REFERENCES}

1. Maki DG. Infections due to infusion therapy. In: Bennett JV Brachmann PS. eds. Hospital infections. 3rd edn. Boston: Little Brown and Co. Inc, 1992:849-98.

2. Maki DG, Kluger DM, Crnich CJ. The risk of bloodstream infection in adults with different intravascular devices: a systematic review of 200 published prospective studies. Mayo Clin Proc 2006;81:1159-71.

3. Maki DG, Rhame FS, Mackel DC, et al. Nationwide epidemic of septicaemia caused by contaminated intravenous products: I. Epidemiologic and clinical features. Am J Med 1976;60:471-85.

4. Millam D. The history of intravenous therapy. J Intraven Nurs 1996;19:5-14. 
5. Band JD, Maki DG. Safety of changing intravenous delivery systems at longer than 24-hour intervals. Ann Intern Med 1979;91:173-8.

6. Eggimann P, Pittett D. Catheter-related infections in the ICU. In: Vincent JL., ed. Yearbook of intensive care and emergency medicine. Springer, 2000:95-110.

7. Josephson A, Gombert ME, Sierra MF, et al. The relationship between intravenous fluid contamination and the frequency of tubing replacement. Infect Control 1985;6:367-70.

8. Rickard CM, Lipman J, Courtney M, et al. Routine changing of intravenous administration sets does not reduce colonization or infection in central venous catheters. Infect Control Hosp Epidemiol 2004;25:650-5.

9. Ullman AJ, Cooke ML, Gillies D, et al. Optimal timing for intravascular administration set replacement. Cochrane Database Syst Rev 2013;9:CD003588

10. Fox M, Molesky M, Van Aerde JE, et al. Changing parenteral nutrition administration sets every $24 \mathrm{~h}$ versus every $48 \mathrm{~h}$ in newborn infants. Can J Gastroenterol 1999;13:147-51.

11. Matlow AG, Kitai I, Kirpalani $\mathrm{H}$, et al. A randomised controlled trial of 72- versus 24-hour intravenous tubing administration set changes in newborns receiving lipid therapy. Infect Control Hosp Epidemiol 1999;20:487-93.

12. Raad I, Hanna HA, Awad A, et al. Optimal frequency of changing intravenous administration sets: is it safe to prolong use beyond 72 hours? Infect Control Hosp Epidemiol 2001;22:136-9.

13. Blight I, Amadio J, Thomas P, et al. Randomised prospective study of replacing intravenous administration sets at 72 hours versus 120 hour intervals in central venous catheters. Conference proceedings of the 4th Nursing Practice Conference. 1998: Adelaide, Australia.

14. Jakobsen CJ, Grabe N, Nielsen E, et al. Contamination of intravascular infusion systems - the effect of changing administration sets. J Hosp Infect 1986;8:217-23.

15. Luskin RL, Weinstein RA, Nathan $\mathrm{C}$, et al. Extended use of disposable pressure transducers. A bacteriologic evaluation. JAMA 1986;255:916-20.

16. Rickard C, Wallis SC, Courtney M, et al. Intravascular administration sets are accurate and in appropriate condition after 7 days of continuous use. An in vitro study. J Adv Nurs 2002;37:330-7.

17. Simon A, Fleischhack G, Wiszniewsky G, et al. Influence of prolonged use of intravenous administration sets in paediatric cancer patients on CVAD-related bloodstream infection rates and hospital resources. Infection 2006;34:258-63.

18. Rickard CM, Vannapraseuth B, McGrail MR, et al. A cross-sectional study investigating the relationship between intravenous infusate colonisation and fluid container hang time. $J$ Clin Nurs 2009;18:3022-8.

19. Marschall J, Mermel LA, Classen D, et al. Strategies to prevent central line-associated bloodstream infections in acute care hospitals. Infect Control Hosp Epidemiol 2008;29(Suppl 1):s22-30.

20. Yokoe DS, Mermel LA, Anderson DJ, et al. A compendium of strategies to prevent healthcare associated infections in acute care hospitals. Infect Control Hosp Epidemiol 2008;29:S12-21.

21. National Health and Medical Research Council in partnership with the Australian Commission on Safety and Quality in Healthcare.
Australian guidelines for the prevention and control of infection in healthcare. Canberra: Department of Health and Aging, 2010.

22. O'Grady NP, Alexander M, Burns LA, et al. Guidelines for the prevention of intravascular catheter-related infections. Clin Infect Dis 2011;52:e162-93.

23. Maki DG. Infections caused by intravascular devices used for infusion therapy: pathogenesis, prevention and management. In: Bisno AL, Waldvogel FA. eds. Infections associated with indwelling medical devices. Washington, DC: ASM Press, 1994:155-205.

24. Ducharme FM, Gauthier M, Lacroix J, et al. Incidence of infection related to arterial catheterization in children: a prospective study. Crit Care Med 1988;16:272-6.

25. O'Malley MK, Rhame FS, Cerra FB, et al. Value of routine pressure monitoring system changes after 72 hours of continuous use. Crit Care Med 1994:22:1424-30.

26. Darouiche RO, Raad I. Prevention of infections associated with vascular catheters. In: Vincent J-L. ed. Yearbook of intensive care and emergency medicine. Berlin: Springer-Verlag, 1998:327-38.

27. deMoissac $\mathrm{D}$, Jensen $\mathrm{L}$. Changing IV administration sets: is 48 versus 24 hours safe for neutropenic patients with cancer. Oncol Nurs Forum 1998;25:907-13.

28. Crump JA, Collignon PJ. Intravascular catheter-associated infections. Eur J Clin Microbiol Infect Dis 2000;19:1-8.

29. Mermel LA, Allon M, Bouza E, et al. Clinical practice guidelines for the diagnosis and management of intravascular catheter-related infection: 2009 Update by the Infectious Diseases Society of America. Clin Infect Dis 2009;49:1-45.

30. Maki DG, Weise CE, Sarafin HW. A semiquantitative culture method for identifying intravenous-catheter-related infection. N Engl J Med 1977;296:1305-9.

31. O'Grady NP, Alexander M, Dellinger EP, et al. Guidelines for the prevention of intravascular catheter-related infections Centers for Disease Control and Prevention. MMWR Recomm Rep 2002;51 (RR-10):1-29.

32. Fortun J. Infections related to intravascular devices used for infusion therapy. Enferm Infecc Microbiol Clin 2008;26:168-74.

33. Macias AE, de Leon SP, Huertas M, et al. Endemic infusate contamination and related bacteremia. Am J Infect Control 2008:36:48-53.

34. Webster J, Clarke S, Paterson D, et al. Routine care of peripheral intravenous catheters versus clinically indicated replacement: randomised controlled trial. BMJ 2008;337:a339.

35. Cercenado E, Ena J, Rodriguez-Creixems M, et al. A conservative procedure for the diagnosis of catheter-related infections. Arch Intern Med 1990;150:1417-20.

36. Cornely OA, Bethe U, Pauls R, et al. Peripheral Teflon catheters: factors determining incidence of phlebitis and duration of cannulation. Infect Control Hosp Epidemiol 2002;23:249-53.

37. Oncu S, Ozsut $\mathrm{H}$, Yildirim A, et al. Central venous catheter related infections: risk factors and the effect of glycopeptide antibiotics. Ann Clin Microbiol Antimicrob 2003;2:3.

38. Tager IB, Ginsberg MB, Ellis SE, et al. An epidemiologic study of the risks associated with peripheral intravenous catheters. $\mathrm{Am} \mathrm{J}$ Epidemiol 1983;118:839-51. 